\title{
Marco conceptual y caracterización del servicio de día para la inclusión social en Bizkaia
}

\section{Óscar Seco Revilla}

Director General de Inclusión Social, Diputación Foral de Bizkaia

oscar.seco@bizkaia.eus

\begin{abstract}
Artikulu honetan, Bizkaiko gizarte-inklusiorako eguneko zentroen esparru kontzeptuala eta karakterizazioa biltzen da, lurralde horretako Foru Aldundiko Gizarte Inklusiorako Zuzendaritzak garatutakoa, Gizarte Zerbitzuetako Zorroko 185/2015 Dekretuan jasotakoa. Artikulu honetan, lehenik eta behin, ereduaren oinarri kontzeptual eta filosofikoak ezartzen dira, gizarte-bazterketaren askotariko kausak aintzat hartuta eta bizi-kalitatea, autodeterminazioa eta integrazio komunitarioa oinarri dituen gizarte-inklusio aktiboa aldentzen duen eredua garatzeko premia. Osagai horiek oinarri hartu ondoren, proposatutako ereduaren ezaugarriak azaltzen dira, eta eskaini beharreko alderdi hauek zehazten dira: prestazio tekniko eta osagarriak, jasotzaileen profilak, zerbitzuaren prestazio kualifikatu gisara ostatua eskaintzea, eta intentsitatearen, iraupenaren edo exijentziaren araberako zerbitzuetako baldintzak.
\end{abstract}

\section{GAKO-HITZAK:}

Eguneko zentroa, gizarte-bazterketa, arreta indibidualizatua, autodeterminazioa, baldintzak, bizi-kalitatea, aktibazio inklusiboa.
Este artículo recoge el marco conceptual y la caracterización del servicio de día para la inclusión social en Bizkaia desarrollado por la Dirección de Inclusión Social de la Diputación Foral de ese territorio, en desarrollo del Decreto 185/2015 de Cartera de Servicios Sociales. El artículo establece en primer lugar los fundamentos conceptuales y filosóficos en los que se asienta el modelo, a partir del reconocimiento del carácter multicausal de la exclusión y la necesidad de desarrollar un modelo de inclusión activa orientado a la calidad de vida, la autodeterminación y la integración comunitaria. A partir de esos elementos, se plantean las características del modelo propuesto y se especifican aspectos tales como las prestaciones técnicas y complementarias que habrán de ofrecerse, los perfiles destinatarios, la provisión del alojamiento como prestación cualificada del servicio y las condiciones de los servicios en cuanto a intensidad, duración o exigencia.

\section{Palabras Clave:}

Centro de día, exclusión social, atención individualizada, autodeterminación, condicionalidad, calidad de vida, activación inclusiva. 


\section{Marco conceptual y modelo de atención}

\subsection{Consideraciones iniciales: servicio público y responsabilidad pública}

El modelo de atención que se propone para el servicio de día para la inclusión social en Bizkaia parte de la constatación de la necesidad de actualizar el paradigma vigente de intervención con las personas en situación de exclusión social, desde el punto de vista de sus bases filosóficas o conceptuales. También parte, como se señala posteriormente, de la necesidad de desarrollar servicios eficientes, estructurados y coherentes, compuestos fundamentalmente por prestaciones técnicas y, adicionalmente, de impulsar el desarrollo de otros tipos de servicios que, si bien no están en el Decreto 185/2015 de Cartera de Servicios Sociales orientados al ámbito de la exclusión social, podrían jugar un papel importante a la hora de materializar el criterio de continuidad en la atención de las personas usuarias en situación o riesgo de exclusión social.

La primera consideración de la que parte este modelo es la de que hemos de dar respuesta a la contingencia de exclusión social (al menos en la vertiente de la que se ocupan los servicios sociales) desde un enfoque de servicio público. Ello implica que hemos de construir un modelo de atención y un mapa de recursos articulado, coherente y homogéneo que apueste por la responsabilidad pública de los servicios bajo los criterios de:

- prioridad: garantizar el acceso a las prestaciones y servicios regulados en el Decreto 185/2015;

- compromiso y esfuerzo por la mejora de la calidad y de los apoyos;

- homogeneidad en la intervención; y

- explicitación de la titularidad pública del servicio: las personas usuarias y la ciudadanía en general tiene que identificar nuestros recursos como recursos públicos del sistema de Servicios Sociales.

En ese sentido, hay que partir de la constatación de que el Decreto 185/2015 obliga a las Administraciones Públicas a garantizar a las personas usuarias una serie de derechos cuya exigibilidad puede hacerse valer tanto en vía jurisdiccional como administrativa. Ello supone, a su vez, una necesaria adaptación de la red de prestaciones y servicios de cara a dar adecuado cumplimiento a las previsiones del citado decreto y, consiguientemente, de los instrumentos reguladores, como es el caso de los convenios de colaboración, máxime teniendo en cuenta la ausencia de un decreto que regule las prestaciones y servicios vinculados a la inclusión social.

Consideramos, por otra parte, que todos los servicios han de partir de un mismo marco conceptual (comprensión de la exclusión social, de los procesos de inclusión y de la metodología de intervención social), de forma que el despliegue de los recursos (tipos de servicio, condiciones de acceso, objetivos de la intervención, perfiles atendidos, prestaciones técnicas preestablecidas), la articulación de los procesos de intervención individual y las normas que deben regir el funcionamiento de los centros y programas de atención directa, respondan a ese marco conceptual, y no a otro.

El marco conceptual propuesto hace también referencia al principio de responsabilidad pública y a su relación con los mecanismos de concertación social. En ese sentido, la indiscutible presencia de la iniciativa social no lucrativa en la provisión y gestión de los servicios públicos ha de asentarse sobre el principio de responsabilidad pública, lo cual requiere asumir el derecho de la persona usuaria a recibir las atenciones señaladas en su plan de atención personalizada mediante las prestaciones descritas en la Cartera de Servicios y específicamente para cada modalidad de centro o servicio. El principio de responsabilidad pública también implica:

- la atribución de la responsabilidad de caso a la persona profesional de la Diputación Foral de Bizkaia o de los servicios sociales de base, según el caso, asignada;

- la coordinación de la/el responsable de caso;

- el reconocimiento y cumplimiento de los derechos y deberes de las personas usuarias y profesionales de los servicios sociales;

- el deber de colaboración con las actuaciones de inspección y control;

- la garantía de una financiación adecuada; y

- la comunicación y visibilización pública de la Administración Pública responsable del servicio o centro.

Igualmente, el Servicio para la Inclusión y las entidades sociales procurarán la adaptación al nuevo modelo de atención, evitando intervenciones, centros o redes paralelas que puedan dificultar la atención a las personas por parte de los servicios públicos. Por otra parte, las entidades sociales solo resultarán de interés especial (solo podrán considerarse proveedoras privilegiadas) si aportan un valor añadido específico y diferencial que se encuentra en la generación de dinámicas comunitarias que sirvan de apoyos complementarios a los propiamente objeto de concertación.

\subsection{Fundamentos básicos del modelo de atención}

Desde el punto de vista de su fundamentación técnica, queremos asentar el modelo de atención sobre cinco fundamentos básicos: concepción multicausal de la exclusión; atención centrada en la persona; calidad de vida y reducción de daños; adaptación de la red, de forma progresiva, al modelo de vida independiente; $y$ enfoque de género. 
En primer lugar, asumimos una concepción multicausal de la exclusión, que responde a múltiples factores y dimensiones, que, a su vez, se interrelacionan entre sí generando procesos dinámicos de expulsión social. En consecuencia, apostamos por desarrollar respuestas institucionales que reconozcan la multidimensionalidad de los procesos de inclusión, poniendo el énfasis en la ciudadanía activa y la activación inclusiva. Ello implica la necesidad de:

- transitar de un modelo rehabilitador o normalizador a un modelo de inclusión que traslada el eje de atención de la persona al entorno social y a la procura de los derechos sociales, a partir de la consideración de que buena parte de los factores de exclusión tienen su origen y solución en lo comunitario;

- la necesidad de que los programas de inclusión trabajen, al margen de la empleabilidad, otras dimensiones vitales como pueden ser el ocio, las actividades culturales, el voluntariado $u$ otras actividades comunitarias o de interés social (de reciprocidad, interdependencia, gratuidad 0 solidaridad), o los desempeños familiares; y

- superar los recursos orientados a la capacitación o la cualificación e impulsar la orientación hacia el empoderamiento y la defensa de derechos, vinculados intrínsecamente con la dignidad de la persona.

En segundo lugar, defendemos un modelo de atención centrada en la persona, que favorezca la individualización de la atención, la autodeterminación y la autonomía de las personas atendidas. Esto implica un tránsito desde un modelo de atención centrado en los centros y recursos a un enfoque comunitario, centrado en las personas, sus prioridades y necesidades y su inclusión social. Estamos, pues, ante un cambio de cultura con respecto al modelo preponderante, que introduce criterios de condicionalidad, de naturaleza moral, en la intervención social con diferentes niveles de “merecimiento" en función de los comportamientos individuales, que no siempre se corresponden con el marco de derecho subjetivo que establece el corpus normativo vigente.

La traslación de este modelo (que proviene del ámbito de la discapacidad) al ámbito de los servicios para la inclusión tiene, al menos, dos implicaciones de importancia:

- por una parte, la calidad de vida - tal y como la experimentan y definen las personas atendidas- se constituye como regla de oro de la intervención, en paralelo al objetivo de inclusión social;

- por otra parte, el modelo de apoyos - estrechamente vinculado a la individualización de la atención- implica un cambio en la forma de concebir la intervención de las/los profesionales, cuya labor ha de centrarse preferentemente en la provisión de apoyos para la mejora de la calidad de vida y para la inclusión social de las personas atendidas.

En esa línea, apostamos en tercer lugar por un modelo orientado hacia la calidad de vida, que aplique, cuando sea necesario, objetivos de reducción de daños. La inclusión social se plantea como el fin de la intervención en este marco. El objetivo básico y prioritario de la intervención ha de ser garantizar la satisfacción de las necesidades básicas y la garantía de unas condiciones mínimas de calidad de vida, en el que se relativicen tanto los requisitos previamente exigibles a las personas como los resultados esperables con ella (conciencia del problema, motivación al cambio, desarrollo de hábitos, puntualidad y cumplimiento de horarios, higiene, abstinencias de consumo de tóxicos, normativas de régimen interior, etc.). En consecuencia, el modelo ha de contemplar una atención de bajo umbral, abriendo la puerta a trabajar con personas que no responden con procesos exigentes o mediante procesos en los que el objetivo esperable sea, simplemente, que la persona sufra el menor deterioro posible, cobrando sentido en este ámbito la intervención social paliativa, la contención y el reconocimiento de la espiralidad de las situaciones de exclusión.

También defendemos la adaptación de la red, de forma progresiva, al modelo de vida independiente. A partir de modelos como la Rehabilitación Basada en la Comunidad o la reforma psiquiátrica, se ha aceptado de forma casi unánime el gradual abandono de las instituciones residenciales y su sustitución por servicios de base domiciliaria o por soluciones residenciales dispersas y de pequeño tamaño, a fin de permitir que las personas en situación de vulnerabilidad puedan vivir con garantías en la comunidad. En el ámbito de la exclusión social, sin embargo, no se ha asumido por completo el cambio de paradigma que implica la apuesta por servicios de base domiciliaria y la necesidad de avanzar hacia modelos de vivienda asistida o con apoyo, en la cual o desde la cual se prestan apoyos individualizados a las personas en situación de exclusión social.

Esta desinstitucionalización o transición hacia la atención basada en la comunidad implica un cambio en el tipo de atención que se presta a las personas con problemáticas asociadas a la exclusión social. Las personas usuarias deben ser valoradas y consultadas, y deben ser atendidas en un espacio alternativo a los grandes equipamientos de tipo institucional, que se adapte a sus necesidades y preferencias, y en el que puedan recibir los apoyos necesarios. No se trata de una serie de cambios puntuales, sino de un proceso que no concluye nunca: no se trata de sacar a la gente de unos edificios llamados “instituciones”, sino de ayudar a las personas a tener, o a recuperar, el control sobre sus vidas y a integrarse en la sociedad, utilizando hasta donde sea posible la misma gama de alojamientos, medidas de convivencia 
y formas de vida que el resto de la población. Se trata, igualmente, de promover que estas personas tengan una buena calidad de vida, participando como ciudadanos de pleno derecho en actividades sociales, culturales y económicas, en la medida y en la forma que elija cada persona.

La atención comunitaria por la que abogamos implica:

- usar un alojamiento situado entre o junto a los alojamientos del resto de la población, que sea adecuado, apropiado y accesible para la persona;

- usar la gama de opciones de alojamiento normalmente disponible para la población en general, desarrollando antes apoyo en la vivienda que viviendas comunitarias o tuteladas con apoyos;

- permitir que las personas, en la mayor medida de lo posible, elijan dónde, con quién y cómo van a vivir; $y$

- prestar toda la ayuda requerida para que las personas participen con éxito en la comunidad.

La materialización más clara de este enfoque es el desarrollo de una red de viviendas de baja exigencia e intensidad de apoyo modulada basadas en el modelo Housing First, cuya puesta en marcha tuvo lugar en nuestro territorio a finales de 2016 a iniciativa del propio Departamento de Empleo, Inclusión Social e Igualdad de la Diputación Foral de Bizkaia. Esta experiencia ha puesto de manifiesto que la baja exigencia es también muy útil para facilitar la implicación de la persona usuaria en procesos más ambiciosos de recuperación personal.

También apostamos por unidades de convivencia, viviendas tuteladas o con apoyos y otros tipos de soluciones residenciales que se caracterizan por su pequeño tamaño, su carácter disperso, la garantía de un marco de privacidad e intimidad, y la prestación de servicios individualizados de apoyo de intensidad variable. En tales casos, la clave del éxito radica en la elaboración de planes individualizados de apoyo y el despliegue de profesionales que implementen esos apoyos y atenciones individualizados y acompañamiento intensivo que pudiesen requerir para posibilitar una vida autónoma (y que contemplen la atención personal y la atención doméstica cuando sea necesaria). En este marco, resulta esencial el aprovechamiento de los apoyos naturales de la persona usuaria, así como los recursos comunitarios que el propio entorno ofrece para la participación de las personas atendidas en actividades culturales, formativas, de ocio y de tiempo libre.

El modelo por el que esta diputación está apostando requiere de un despliegue progresivo y paulatino, a la vez que la transformación de la actual red existente. Esta transformación se desarrolla mediante los siguientes pasos:
- Adaptación de las condiciones de los centros existentes en cuanto a intensidad, duración o exigencia:

- la intensidad, medida por la dedicación necesaria de los apoyos técnicos que se precisan, a través de los instrumentos de valoración especializada con que contamos.

- la exigencia, posibilitando una intervención de bajo umbral que surge como una necesidad de no crear excluidos dentro de los procesos de inclusión, ya que nos encontramos con un grupo importante de personas que inician procesos una y otra vez y que no se adecuan a los perfiles prediseñados por los centros.

- la duración, estableciendo con carácter general que los procesos de inclusión social son temporales, procurando evitar cronicidades y que se generen situaciones de dependencia (aunque tendremos que mantener la consideración de crónicos para personas muy dañadas y de edad avanzada que requieren altos niveles asistenciales y probablemente irreversibles).

- Priorización de la perspectiva relacional (acompañamiento social, coordinación de caso) y de los objetivos de intervención orientados a la mejora de la calidad de vida y a la autodeterminación de las personas usuarias, especialmente en aquellos dispositivos dirigidos $a$ atender a personas en situación de cronicidad o de mayor vulnerabilidad.

- Refuerzo de la intervención psicosocial (prestación menos desarrollada, hasta el momento) en todos los servicios.

- Énfasis en la centralidad de las personas y sus derechos (tanto en los reconocibles como derechos sociales, como en los que se derivan de su condición de administrado), que han de tutelarse más allá de las necesidades organizacionales y los criterios profesionales.

Consideramos finalmente la necesidad de aplicar un enfoque de género a la definición de todos los servicios. Unida a la comprensión multidimensional de la exclusión social, se encuentra la existencia de una brecha de género en el ámbito de la exclusión. Las mujeres se encuentran comparativamente en peor situación social que los hombres; también podemos decir que cursa de forma distinta y con itinerarios de exclusión diferentes al de los hombres, que se plasman en la mayor invisibilidad social de la alta exclusión social femenina. Las mujeres suelen tener vínculos, normalmente de dominación machista, a través de los que evitan espacios especialmente hostiles, o disponen de empleos, como trabajadoras sexuales o en redes de explotación, que ocultan parcialmente esta realidad. Diversos autores ponen de manifiesto que, ante situaciones similares de exclusión social, las mujeres sufren comparativamente situaciones de mayor deterioro físico y mental y acumulan mayores problemáticas, entre ellas, la de sufrir violencia de género. 
Desde este punto de vista, las estrategias para hacerles frente también han de ser distintas y afectan a la configuración de los recursos, a la tipología y formación de los profesionales, a la incorporación de consideraciones específicas de desprotección y riesgo, etc. Por ello, la necesidad de abordar, en un contexto eminentemente preventivo, intervenciones psicosocioeducativas en el ámbito social y en servicios predominantemente mixtos, nos obliga a:

- desarrollar una actitud de desenmascaramiento de las desigualdades por razón de género (que se expresan en diferentes factores psicosociales, patrones de conducta, en las diferentes interacciones humanas - tanto en la vertiente relacional como en la emocional-, en la invisibilidad de las necesidades específicas de las jóvenes, en la mirada androcéntrica de buena parte de las aproximaciones científicas en el ámbito de la intervención, en las desigualdades efectivas en derechos y oportunidades, etc.;

- desarrollar mecanismos y protocolos de prevención y atención a las diferentes modalidades de violencia contra la mujer que se expresan también en este ámbito;

- elaborar itinerarios orientados al empoderamiento de la mujer (atribución y fortalecimiento de derechos, ejercicio de estos, detección y superación de vulnerabilidades, atenciones diferenciadas cuando sea conveniente) y superación de roles de género que marcan mucho en los procesos (por ejemplo, la vinculación de la masculinidad con la vida social o los recursos para el empleo, o, en la vertiente femenina, las expectativas y la responsabilización vinculada con lo familiar);

- desarrollar estrategias diferenciadas. Los recursos y su configuración han de tener en cuenta esta perspectiva (sin incurrir en planteamientos sexistas), desarrollando espacios seguros y acogedores. Se ha de contemplar la interseccionalidad (de las diferentes vulnerabilidades exclusión-juventud-mujer) y la integralidad de la intervención;

- fomentar que las personas profesionales tengan formación específica en igualdad y violencia contra la mujer; y

- en el ámbito de los programas que trabajen con varones, se han de desarrollar modelos de trabajo sobre nuevas masculinidades y abordar específicamente las situaciones de violencia hacia la mujer, en todas sus modalidades.

En consecuencia, los dispositivos y servicios que se desarrollen han de estar prioritariamente centrados en las mujeres, han de ser capaces de diseñar actuaciones de inclusión que consideren la perspectiva de género, evitando dobles victimizaciones y espacios hostiles, $y$ desarrollando una atención integral que considere su empoderamiento, también, como mujer. En esa misma línea, es exigible una especialización en el trabajo con mujeres, la preocupación por la relación entre la condición de mujer y su relación con la adicción, la exclusión social o residencial, así como poner el punto de mira en la atención a las/os hijas/ os e integrando en la intervención el rol de madre.

\subsection{Consecuencias del cambio ideológico y características del nuevo modelo}

Las consecuencias de la adopción del marco conceptual enunciado son muy variadas. En primer lugar, hemos de repensar la "motivación al cambio" como requisito de acceso. No puede establecerse como condición de acceso a un servicio que la persona asuma la necesidad de operar en su vida grandes transformaciones, porque muy probablemente no sea ni tan siquiera consciente de tener un problema distinto al de las necesidades sentidas. En lo sucesivo, nos resultará suficiente que la persona excluida acepte la figura profesional y realice una demanda de reducción de daños. La toma de conciencia del problema y la necesidad de construir un plan de trabajo gobernable por el propio sujeto forman parte de estadios más tardíos de la intervención.

En segundo lugar, tenemos que optar por un modelo de baja exigencia (en cuanto a requisitos de acceso y permanencia) y de objetivos intermedios (reducción de daños), y abandonar el modelo terapéutico o rehabilitador, que responde a una intervención moralizante y finalista centrada en la idea del merecimiento y la adecuación a patrones estándar de comportamiento. La expulsión del servicio no es solución ni instrumento de intervención. El servicio ha de estar orientado a la solución, no a la sanción.

Debemos también replantear los objetivos de la intervención. La autodeterminación, entendida como dotar a la persona del apoyo que precisa para que esté en condiciones de tomar sus propias decisiones y, en consecuencia, adoptar el estilo de vida que desea dentro de su comunidad, y no tanto en persuadir para la modificación de conductas. Esto tiene su correlato en el concepto de acompañamiento, que no es fiscalizador ni controlador de conductas, sino una relación proactiva, "una alianza dinámica entre la persona y los y las profesionales que le atienden en torno a un proyecto común", según se define en el manual del proyecto INCLUE. De esta manera, son las personas profesionales las que se adaptan a las usuarias y no estas a las normas de régimen del centro.

Además, debemos ser capaces de basarnos en un modelo de apoyos. Los apoyos se entienden como recursos y estrategias, diversificadas por su contenido y variables en el alcance e intensidad, que facilitan el desenvolvimiento exitoso de una persona en los diferentes contextos vitales, promoviendo el logro de metas valiosas. En ese sentido, la opción por la Planificación Centrada en la Persona (PCP) conlleva sustituir modelos de atención agrupados, 
tipo clúster (racimo) de atención, en favor de un modelo de apoyos profesionales individualizados que permita equilibrar los que la persona quiere, al ritmo que quiere, con lo que la persona puede y lo que necesita.

Para ello precisamos de recursos flexibles y adaptados, que integren en el proceso de intervención los apoyos naturales de la persona (familiares y comunitarios). Esto exige:

- adaptar la intensidad de la atención, de forma inversamente proporcional a los recursos que la persona muestra para afrontar los objetivos propuestos;

- considerar que la persona está antes que el recurso: el lugar en el que se desenvuelve el proceso es el natural de la persona, y el apoyo individualizado antes que el programa de actividades colectivas del servicio;

- no imponer o exigir la realización de actividades grupales o convivenciales, particularmente para aquellas personas que rechazan o tienen dificultades relevantes de socialización;

- desarrollar modelos de intervención en medio abierto y de apoyo a la vida independiente que posibiliten el desenvolvimiento de la persona usuaria en sus ámbitos naturales (entorno y domicilio, principalmente) con prestaciones técnicas que contengan atención personal y atención doméstica y reforzando procesos de acompañamiento personal no necesariamente vinculados a actividades de tipo congregado;

- asumir la idea de que el empleo no es, en sí mismo o por sí solo, suficiente para garantizar la inclusión; de hecho, puede igualmente pensarse que en determinados casos o situaciones los procesos de inclusión no requieren necesariamente de la inclusión laboral y que pueden existir otras dimensiones vitales sobre las que resulta prioritario trabajar; $y$

- adecuar los servicios para que brinden una atención centrada en la persona antes que en el recurso. El recurso es en todo caso un medio para conseguir un fin -la inclusión social-y no un fin en sí mismo. Nadie logra una inclusión social plena por estar perfectamente adaptado a las normas de uno u otro recurso. Desde esta óptica, es el recurso el que debe adaptarse a las necesidades de cada persona y no al contrario. Esto exige introducir progresivamente modelos de apoyo itinerante y domiciliario y en medio abierto. A nuestro juicio, una atención de base comunitaria ha de incidir especialmente en el refuerzo de las prestaciones técnicas en los servicios de día que se han de desplegar en mayor proximidad, adecuando los apoyos residenciales a los casos necesarios y por tiempos reducidos, y evitando el riesgo de la institucionalización.

\section{Caracterización del servicio de día para la inclusión social}

\subsection{Delimitación de los servicios de atención primaria y secundaria}

En virtud del artículo 5.3 del Decreto $185 / 2015$, las diputaciones forales serán competentes para la provisión de los servicios de atención secundaria del Catálogo de Prestaciones y Servicios del Sistema Vasco de Servicios Sociales. Entre dichos servicios figura el servicio o centro de día para atender necesidades de inclusión social, cuya regulación se contiene en la Ficha 2.2.3 del Anexo I del mencionado Decreto. Este servicio ofrece a las personas usuarias un conjunto de prestaciones técnicas, de carácter polivalente, orientadas a su proceso de inclusión e incorporación social, procurando que no se desliguen de su entorno habitual y ofreciéndoles un acompañamiento individualizado en su itinerario de inclusión.

La definición de este servicio exige, en primer lugar, aclarar la delimitación entre la atención primaria y la secundaria. Como se sabe, el catálogo de servicios de la Ley 12/2008 asigna, en general, los servicios considerados como de atención primaria a los ayuntamientos y los considerados de atención secundaria a las diputaciones forales. Si bien en algunos casos el servicio prestado corresponde a un único nivel competencial, en muchos otros se establece un sistema doble, en función del cual el mismo servicio corresponde a los dos niveles institucionales, diferenciándose en lo que se refiere a la intensidad de la atención, los objetivos de la intervención, el nivel de exigencia o la población potencialmente atendida. Si bien en otros ámbitos de los servicios sociales esta delimitación de funciones ha podido resultar adecuada, en el caso de la exclusión social introduce una cierta confusión, que se agrava si se tienen en cuenta algunos de los problemas que el Decreto de Cartera presenta en este ámbito (ausencia de criterios claros para diferenciar operativamente el contenido de determinados centros o servicios de atención primaria o secundaria, no inclusión en el decreto como potenciales usuarios de algunos servicios o centros de las personas en situación de exclusión o en situación categorizada de riesgo de exclusión, etc.).

En el caso del centro de día al que nos referimos, esta disquisición resulta relevante porque puede colisionar competencialmente con la Ficha 1.7.2, "Centro de atención diurna para personas en situación de exclusión social”, orientada hacia el mismo perfil de población - personas en situación de exclusión social- con similitud de funciones en algunos casos y que puede generar solapamientos y déficits en el continuo de atención.

El Decreto de Cartera plantea una serie de elementos diferenciadores de los diversos tipos de centros, en los que se combinan -de forma no 
siempre sistemática- criterios relacionados con, al menos, los siguientes elementos: intensidad de la atención, duración de la estancia, perfil de las personas usuarias, nivel de exigencia, tipo de acceso y naturaleza del recurso. De acuerdo a lo que establece el propio decreto, el criterio básico que debe delimitar los servicios de atención primaria y los de secundaria es el referido a la intensidad de la atención. En ese sentido, el denominador común de los recursos de atención primaria habrá de ser la baja intensidad (y no la situación de la persona en el eje que va de la inclusión a la exclusión, ni el nivel de exigencia, ni la duración de la estancia), mientras que el denominador común de los recursos de atención secundaria será la media y alta intensidad. Estos criterios podrán después combinarse - de acuerdo al Decreto de Cartera y a los acuerdos específicos que se establezcan en este proceso de planificacióncon diferentes posibilidades en lo que se refiere a la duración de la estancia, el perfil de las personas usuarias, el nivel de exigencia, el tipo de acceso o la naturaleza del recurso.

En su consecuencia, es la afectación al criterio uno de la herramienta de valoración de la exclusión -la capacidad de resistencia de la persona, que requiere cuando menos de apoyos de media intensidad-, el que establece el linde competencial interno de la diputación foral. Este criterio se adecua al criterio establecido por la Dirección General de Inclusión de la Diputación Foral de Bizkaia en octubre de $\mathbf{2 0 1 5}$, que da entrada a la intervención social con personas en situación diagnóstica de riesgo de exclusión social por criterio uno, considerando que no puede ser discriminante competencial la categoría diagnóstica (la desafortunada y errónea inferencia del artículo 27 de la Ley 12/2008 de Servicios Sociales, en el sentido de que la exclusión es competencia foral y el riesgo competencia municipal).

\subsection{El servicio de día de atención secundaria para la inclusión social: prestaciones técnicas y complementarias}

El Decreto de Cartera establece como servicio paradigmático de intervención en el ámbito de la exclusión social bajo los parámetros de proximidad y baja exigencia (presupuestos de nuestro marco conceptual e ideológico) el servicio (o centro) de día, que responde según la Ficha 2.2.3. a un equipamiento "de proximidad, flexible y abierto durante un amplio abanico de horas de atención diurna, cuyo objetivo es dotar a la persona del máximo grado de autonomía y favorecer su inclusión social. Es un servicio de media y alta intensidad de apoyo y de baja, media o alta exigencia”. Es esta precisamente nuestra opción por una intervención comunitaria, próxima, altamente cualificada y de elevada intensidad y multiplicidad de apoyos, que además requiere los siguientes elementos:

- Una intervención desperfilada. La clasificación tradicional por colectivos, desde este prisma, carece de sentido: inmigrantes, exreclusos, toxicodependientes, patología dual, VIH, personas sin hogar, enfermedad mental, etc., se nos antoja una segmentación (para la intervención social desde el parámetro de la inclusión social) injustificada y exclusógena.

- Complementariedad de servicios y prestaciones, a partir de la idea del servicio de día. El servicio de día es un servicio social y, de acuerdo con el artículo 12.6 del Decreto 185/2015, se configura como un conjunto de apoyos formales que podrán compatibilizarse entre sí a fin de ofrecer un apoyo integral y adaptar la intervención a las necesidades, capacidades y, en lo posible, preferencias de cada persona, así como a las características de su contexto. Asimismo, en la determinación del recurso, o combinación de recursos más idónea, y en coherencia con el modelo comunitario, se adoptarán enfoques de prevención y promoción de la autonomía, y participación y calidad de vida en la comunidad. En ese sentido, creemos que el servicio de día no ha de concebirse como "un lugar en el que pasar el día”, según la redacción del Decreto de Cartera, soportado en profesionales con mera finalidad presencial y de bajo perfil. Al contrario, ha de definirse más bien como un "servicio orientado a su incorporación social, que ofrece un acompañamiento individualizado en el itinerario de inclusión con intervenciones de carácter ocupacional, educativas, terapéuticas y de inclusión sociolaboral" de alto valor añadido que ofrece prestaciones técnicas (al menos las previstas en el Decreto de Cartera).

Con la finalidad de remarcar esta apuesta, y a sabiendas de que el Decreto de Cartera ofrece ambas denominaciones (servicio o centro), optamos por el primero para romper con la referencia arquitectónica y cerrada del segundo, ya que muchas de las intervenciones habrán de discurrir extramuros de él. No es el recinto el centro de la intervención, sino la persona.

- Provisión de prestaciones técnicas (medidas por su intensidad, esto es, como cantidad de prestación profesional por persona y periodo de tiempo) con arreglo al Decreto de Cartera. Una intensidad adaptada a las necesidades que hoy en día se nos ponen de manifiesto supone, en la práctica generalidad de los recursos con que contamos, una significativa ampliación de las prestaciones técnicas, con mayor alcance y adaptadas a las necesidades que se requieren para la inclusión social. Las que menciona la ficha correspondiente son las siguientes:

1. Acompañamiento social, facilitando apoyo instrumental, emocional, educativo y relacional a las personas usuarias.

2. Intervención socioeducativa y psicosocial, que comprenderá:

- Intervención psicosocial, con el objetivo de propiciar la modificación y mejora de las situaciones y contextos del entorno de las 
personas. Propiciar y promover cambios en los repertorios conductuales de las personas, así como en las condiciones familiares o comunitarias, dirigidos a reducir y/o eliminar los factores que crean o mantienen una situación de vulnerabilidad o dificultad social.

- Intervención educativa o socioeducativa: las personas usuarias, mediante la relación educativa con una/un profesional, modifican actitudes y hábitos inadecuados y adquieren actitudes y hábitos adecuados para el desenvolvimiento autónomo e integración social en cualquier contexto. Podrá desarrollarse a nivel individual, familiar, grupal y/o comunitario y en diversos contextos: el centro, el domicilio familiar y el entorno comunitario. Se incluyen en ella, entre otras posibles, las actividades de ocio educativo, educación de calle, educación familiar, educación doméstica y orientación a personas y familias en situación de crisis. Esta intervención tendrá una función preventiva que conlleva generar mecanismos para la detección de la población destinataria en los contextos en los que se desenvuelve, identificando necesidades y carencias en los contextos en los que aparecen, desarrollando acciones de prevención.

- Intervención ocupacional o prelaboral: prestación en virtud de la cual las personas usuarias, mediante la participación en actividades de capacitación, adquieren o desarrollan actitudes, conocimientos y habilidades útiles para su desenvolvimiento autónomo e integración social, susceptibles de ser aplicadas en el ámbito laboral (preparación para el empleo). Las actividades de capacitación (habilitación) integral persiguen el desarrollo y mejora de habilidades adaptativas relacionadas con el ámbito laboral pero no exclusivas de él, buscando el desarrollo de la autonomía personal y aquellos progresos que favorezcan la inclusión integral de las personas usuarias

- Intervención estimulativa o rehabilitadora: proporcionar apoyo o estímulo para adquirir, desarrollar, conservar o recuperar actitudes, habilidades o capacidades físicas, cognitivas o conductualesinstrumentales necesarias para posibilitar mantener o adquirir el máximo grado de autonomía.

3. Mediación-intermediación, en virtud de la cual se orientará a la persona al desarrollo de sus competencias sociales, favoreciendo la mejora de la interacción entre la persona y en el medio familiar, grupal o comunitario en el que se desenvuelva. Comprenderá, entre otras, las actividades siguientes:
- Promover las redes sociales de apoyo para la incorporación social de las personas en situación de exclusión social fomentar su incorporación a asociaciones, promocionar su asociacionismo, su participación en la vida y en el entorno local, fomentar redes de amistad, vecinales, etc.).

- Favorecer la receptividad de diferentes agentes sociales (escuela, empresa, vecindario, comercio, ayuntamiento) a la incorporación social de los colectivos con dificultades sociales transmitiendo una información adecuada y una visión positiva de ellos, y fomentando valores de solidaridad y cooperación social.

- Intervenir con la familia en los casos en los que se valore que la persona pueda volver a retomar contacto con ella y esta así lo desee.

- Valoración de seguimiento.

- Provisión de prestaciones complementarias. En idéntico sentido, de conformidad con lo recogido en el Decreto $185 / 2015$, deberán garantizarse las prestaciones complementarias y/o accesorias siguientes:

1. Manutención, limpieza, consigna, higiene personal y lavandería.

2. Prestaciones de salud, en su caso, o prestaciones propias del ámbito de los servicios sociales, en tanto que son complementación y promoción de la autonomía funcional de la persona para actividades de la vida diaria, que podrán comprender:

- Dispensación de medicamentos bajo prescripción médica.

- Control y seguimiento de las pautas y tratamientos médicos señalados.

- Acompañamiento a consultas o pruebas médicas.

- Coordinación con los centros de salud pertinentes.

- Incorporación a nuestro modelo de otras prestaciones técnicas no incluidas en el Decreto de Cartera para esta tipología de servicio. Cabe pensar que las posibilidades de intervención social en un proceso de inclusión no se agotan en las prestaciones antes señaladas, lo que obliga a plantear la posibilidad de incorporar al modelo otros servicios de la cartera, abriéndolos a personas en situación o riesgo de exclusión: el servicio de apoyo a la vida independiente (2.7.2.1) y el servicio de intervención socioeducativa y/o psicosocial con familias (2.7.3.1), de forma que se garanticen los siguientes puntos:

1. Una intervención socioeducativa $y / 0$ psicosocial con familia, que comprenderá un conjunto de prestaciones relacionales orientadas a apoyar a unidades familiares o convivenciales de la persona usuaria que 
recibe acompañamiento en el marco del servicio convenido, al objeto de:

- desarrollar apoyos eficientes encaminados a sustituir hábitos, comportamiento, percepciones, sentimientos y actitudes inadecuados, por otros más adaptados, así como a adquirir o a desarrollar las capacidades necesarias (actitudes, conocimientos, criterios, pautas, habilidades) para hacer posible una adecuada convivencia y/o el cuidado adecuado de las personas que se encuentran a su cargo, modificando, en su caso, aquellos aspectos que dificulten un adecuado ejercicio de las funciones y responsabilidades parentales, familiares y convivenciales; $y$

- prevenir un mayor deterioro del contexto convivencial, procurando la contención y el abordaje de comportamientos inadaptados o de situaciones de crisis.

2. Apoyo a la vida independiente, que se articulará a partir de un programa de vida independiente a desarrollar por el servicio de día, dirigido a personas en situación de exclusión social, que residen en domicilios particulares y que tienen necesidades de apoyo para su desenvolvimiento autónomo y su participación activa en el entorno comunitario. Consistirá en un conjunto organizado y coordinado de acciones de acompañamiento que les ofrece apoyo en su proceso de inclusión de vida independiente, de una manera dinámica, flexible y adaptada a las necesidades de cada persona. Las prestaciones técnicas que esta apuesta requiere, son además de las que son características de los centros de día para la exclusión social:

- Atención personal: prestación en virtud de la cual las personas usuarias reciben ayuda, parcial o total, para realizar actividades básicas de la vida diaria necesarias para su cuidado personal, en particular para levantarse y acostarse, asearse, desplazarse, comer $\mathrm{u}$ otras funciones básicas o actividades instrumentales que las vinculen con su entorno, en particular, para comunicarse y acceder al entorno familiar y comunitario. Igualmente, incluyendo, en su caso, el entrenamiento en las habilidades necesarias para realizarlas.

- Atención doméstica: prestación en virtud de la cual las personas usuarias reciben ayuda, parcial o total, para realizar las actividades instrumentales de la vida diaria, en particular las tareas domésticas relacionadas con la alimentación, el vestido y la limpieza de mantenimiento de las instalaciones de la vivienda donde se desarrolla su vida, facilitando su organización, incluyendo, en su caso, el entrenamiento en las habilidades necesarias para la realización de dichas tareas.

3. La necesidad de desarrollar en cada servicio de día planteamientos de intervención en medio abierto, específicamente orientados a las personas en situación de exclusión social grave y, particularmente, a aquellas que carecen de domicilio, así como de reforzar funciones de acompañamiento individual de carácter intensivo, a partir preferentemente del modelo de vida independiente, que nos permita consolidar la opción de disociar la provisión de los servicios de alojamiento de la provisión de los servicios de acompañamiento individualizado.

Algunos de los elementos señalados anteriormente implican una cierta ampliación de los derechos que se reconocen a la ciudadanía a través del mapa y del Decreto de Cartera. La adopción de criterios que pueden ir más allá de esa definición de derechos - por poner un ejemplo, el desarrollo de un programa de apoyo a la vida independiente para personas en situación de exclusión social, la creación de plazas en centros ocupacionales para personas en situación de exclusión social o el desarrollo de una red de viviendas de inclusión de alta intensidad y baja exigencia que respondan a la metodología Housing First- no implica en cualquier caso que tales servicios se entiendan como prestaciones sujetas al derecho subjetivo que reconoce la Ley de Servicios Sociales, sino como servicios que la Dirección de Inclusión Social desarrolla de manera complementaria (en ocasiones, a título experimental) de forma adicional a las obligaciones que se establecen en el Decreto de Cartera y el Mapa de Servicios Sociales.

- Eventualmente, se plantea como necesario desarrollar un sistema de prestaciones económicas, supletorio al de garantía de ingresos, que permitan a la persona usuaria mayores cotas de autonomía, orientadas a garantizar la supervivencia material y la tenencia de recursos de alojamiento autónomo en el caso de que la persona pueda sostenerse en él con los apoyos necesarios, de forma que contribuya a la mayor eficiencia del sistema.

\section{La articulación del servicio en el conjunto de la red: perfiles, provisión de alojamiento y distribución territorial}

\subsection{Perfiles destinatarios del servicio de día}

La definición del modelo de atención a la exclusión social por la que ha optado la Dirección de Inclusión Social (orientado a la calidad de vida e individualización de los apoyos) implica ampliar el 
acceso a determinados servicios - y en especial a los servicios de día- a individuos o perfiles abiertos y heterogéneos de exclusión social, toda vez que contemplamos la realización de intervenciones en buena medida individuales o personales (si bien teniendo en cuenta que el foco se proyecta sobre su medio comunitario, no necesariamente sobre la realidad congregada y plural que el servicio de día representa).

En especial, se considera preciso flexibilizar los requisitos de necesidad si lo que se quiere es desarrollar una mayor capacidad del sistema para desarrollar los apoyos que verdaderamente requieren las personas para su inclusión social. Y, en lo que atañe a los requisitos administrativos, se considera conveniente desbordarlos cuando estos (como en el caso de la antigüedad en el empadronamiento o la situación administrativa) se convierten en concausa de exclusión o marginación que agrave o prolongue esta situación.

Igualmente, nos interesa distinguir entre valoración de la situación de exclusión social, que nos ofrecerá una categoría diagnóstica resultante (solo la situación de exclusión social genera derecho subjetivo a alguno o varios de los apoyos pertinentes al nivel de responsabilidad foral) y diagnóstico, que nos ofrecerá el programa de intervención más adecuado y los niveles de intensidad requeridos para las personas objeto de atención. Recordamos de nuevo aquí que un nivel de intensidad medio o alto será responsabilidad foral y, por tanto, la intervención con personas en riesgo de exclusión que precisen de niveles de apoyo de media o alta intensidad corresponderá a este nivel competencial, si bien sin garantía de derecho subjetivo ni gratuidad de los servicios que se dispensen.

La edad es otro de los requisitos de delimitación. La ficha del Decreto de Cartera menciona los comprendidos entre los 18 y los 64 años. Es evidente que este límite superior resulta incongruente con el previsto en la Ficha 2.4.5. ("Servicios residenciales para la inclusión social"), que no establece techo de edad, por lo que personas en situación de exclusión social de más de 64 años solo podrían ser atendidas en centro residencial (lo que resulta incoherente con los principios de proximidad y enfoque comunitario e individualización antes descritos). En consecuencia, no se establecerá ningún tope de edad para recibir los apoyos que cabe brindar desde un servicio de día.

Por último, ha de mencionarse todo lo relativo a la exigencia contemplada en los requisitos de acceso que nos hará releer y replantear todos ellos, y particularmente los que hacen referencia a la conciencia, motivación y compromiso en relación con el proceso de inclusión o a los requerimientos de convivencia, entendiéndose como tales la capacidad de las personas usuarias de solicitar y aceptar ayuda e intervención profesional, y cuyo objetivo al menos sea detener un proceso desocializador u obtener una asistencia paliativa, sin exigir participación organizativa, colectiva o convivencial cuando las propias personas no están en condiciones de resistir esa dimensión.

\subsection{La provisión del alojamiento como prestación cualificada del servicio de día}

Nuestra posición en relación con la provisión de recursos residenciales (Fichas 2.4.5. y 2.4.6.) parte de la consideración de que el impacto que, en general, tienen en la población atendida es “descontextualizante", tanto porque saca a la persona de su entorno vital y geográfico, porque dificulta los apoyos naturales y porque implica una "convivencia terapéutica" forzada. El enfoque que defendemos, por el contrario, comprende una inclusión en la que tienen mucho que ver los recursos de base comunitaria, normalizados y no estigmatizantes que favorezcan el empoderamiento de la persona usuaria, su participación y atribución de derechos subjetivos, también los que le corresponden del sistema de vivienda.

Sentado lo anterior, nuestra premisa es la de no atender desde soportes artificiales cuando cabe otra intervención menos "lesiva" desde la óptica de la inclusión social de cada persona usuaria. En consecuencia, la atención central de la persona (el grueso de la intervención social) ha de pivotar sobre el servicio de día, en el que se ofrecerán todas las prestaciones técnicas con las intensidades graduables que cada persona precise. El apoyo residencial, entendido en todo caso como complementario, se ha de ofrecer únicamente para procesos en que la persona no se pueda sostener por si misma ni siquiera con apoyos o cuando existen razones que aconsejen adecuada una atención residencial congregada o precisen de otros apoyos (por ejemplo, seguridad en el caso de víctimas de violencia machista). En todos los demás casos, corresponde a los servicios de día la gestión de viviendas y alojamientos normalizados que sirvan de apoyo a los referidos procesos (en forma de atención en el propio domicilio, en viviendas compartidas, metodología Housing First integrada en cada servicio de día, pensiones sociales o cualesquiera otras modalidades de alojamiento) incluyendo la mediación en la convivencia y el apoyo, supervisión y control de la aportación económica que las personas usuarias deban realizar para hacer frente a los gastos de las citadas viviendas.

Como prestación adicional concebimos una prestación técnica de atención doméstica, de forma que las personas usuarias que no tienen plaza residencial (diferenciada del alojamiento con apoyos) puedan "recibir ayuda, parcial o total, para realizar las actividades instrumentales de la vida diaria, en particular, las tareas domésticas relacionadas con la alimentación, el vestido y la limpieza de mantenimiento de las instalaciones de la vivienda donde se desarrolla su vida, facilitando su organización, incluyendo, en su caso, el 
entrenamiento en las habilidades necesarias para la realización de dichas tareas".

En coherencia con lo anterior, el esperable tránsito entre recursos residenciales o de alojamiento, previsiblemente en lógica de mayor autonomía, no supone quiebras de los procesos ni procesos de revinculación, porque la referencia educativa se encuentra, en última instancia, garantizada desde el servicio de día asignado. De esta manera, ponemos nuevamente en el centro de la actividad del servicio de día la regeneración de vínculos primarios (familiares y comunitarios) que no deben suplirse por las personas con quienes comparten dispositivos y recursos.

\subsection{Condiciones de los servicios en cuanto a intensidad, duración o exigencia}

El proceso de definición del modelo de atención, del que debe derivar el Mapa de Servicios Sociales para la inclusión, requiere también reflexionar sobre algunas de las condiciones que se atribuyen a los diversos centros, especialmente en lo que se refiere al nivel de exigencia, la duración de la estancia o la intensidad de la atención. Dentro del proceso de replanteamiento del servicio para la inclusión, hemos apostado por servicios diversificados en los que se combinen los elementos que se han establecido, con abordajes multidisciplinares y recursos que hibriden las diferentes prestaciones técnicas definidas en el Decreto de Cartera.

Estos servicios vienen a procurar una atención temporal a las personas usuarias, que se concretaría en un planteamiento de una intervención limitada en el tiempo con carácter revisable de cara a su prórroga, finalización o cambio de nivel de intensidad. Así, el servicio se organizará para poder ofrecer diferentes niveles de intensidad de atención. Del mismo modo, se planteará un itinerario de intervención cuyo desarrollo evidenciará el progreso por hitos e indicadores objetivables que se concretarán en la formulación y reformulación de la demanda de cada caso y en la evaluación de cada PIA y contarán con un espacio articulado de intervención con las familias y/o realidades convivenciales que presenten que apuesta por un contenido educativo-terapéutico.
3.4. Servicios adaptados a los grados de proximidad contemplados en el mapa

Según la ordenación territorial aprobada, los servicios de día de atención secundaria para la inclusión se han de desplegar en el ámbito geográfico de sector (agregación de comarcas, con un ámbito poblacional comprendido entre $\mathbf{2 0 0 . 0 0 0}$ y 400.000 habitantes). De cara a ir profundizando en el despliegue requerido por el mapa, abogamos por modelos que permitan garantizar la continuidad de la atención entre la primaria y la secundaria a través de un mismo centro más o menos polivalente que permita articular niveles variables de intensidad y exigencia, y diferentes combinaciones de prestaciones técnicas, en función de las necesidades concretas de cada persona, tratando de soslayar los problemas derivados de la difícil coordinación interinstitucional. Del mismo modo, los servicios han de ir creciendo en mirada intersectorial: en relación con el sistema de salud (en especial salud mental), de vivienda, de empleo, o educativo (EPA, etc.).

\subsection{Algunos elementos adicionales a considerar en los nuevos recursos}

Junto a los elementos señalados, es también fundamental tener en cuenta algunos elementos adicionales en la definición de los nuevos recursos:

- Robustecer la coordinación de caso y el papel del profesional de referencia en los procesos de inclusión social. Hasta ahora no todos los servicios de día cuentan con este apoyo.

- El papel del voluntariado, siendo importante, se orienta a desarrollar las funciones de carácter interrelacional y desarrollo de lazos comunitarios más informales, sin que pueda suplir ninguna de las prestaciones técnicas que se han de prestar necesariamente por profesionales titulados en áreas de intervención social.

- Desarrollar un enfoque de género, que atienda la relación entre la condición de mujer y la exclusión social.

- Reforzar la intervención psicosocial como prestación técnica preceptiva en los recursos de inclusión social. 


\section{Bibliografía referenciada}

ARARTEKO (2016): La situación de los servicios sociales municipales en la Comunidad Autónoma de Euskadi. Situación actual y propuestas de mejora, Serie Informes Extraordinarios, VitoriaGasteiz, Ararteko.

BLANCO, J. et al. (coords.) (2015): Modelo Inclúe. La apuesta por una atención de calidad en el ámbito de la inclusión social, Vigo, Grupo Emaús Fundación Social.

FANTOVA, F. (2017): "Servicios Sociales e inclusión social: análisis y perspectivas en el País Vasco",
Zerbitzuan, n- 64, págs. 9-31, 〈https://doi. org/10.5569/1134-7147.64.011.

SIIS CENTRO DE DOCUMENTACIÓN Y ESTUDIOS (2015):

"Reordenación de la red de recursos y programas para la inclusión social de Gipuzkoa”, Zerbitzuan, nํ5ㄹ, págs. 5-38, 〈https://doi.org/10.5569/1134-7147.59.01〉.

ZALAKAIN, J. (2017): "Sistemas de bienestar y sociedad inclusiva: de la inclusión activa a la activación inclusiva”, Documentación Social, nํ186, págs. 173-189. 